\title{
Pharmaceutical Field Encloses a Wide Number of Themes from Drug Discovery
}

\author{
Fizza Batool* \\ Research Officer in Civil Society Association of Pakistan, Pakistan \\ *Corresponding author: Fizza Batool, Research Officer in Civil Society Association of \\ Pakistan, Pakistan, Tel: +92347518695; Email: fizzabatool90@yahoo.com
}

\section{Mini Review \\ Volume 3 Issue 4}

Received Date: October 01, 2018

Published Date: November 12, 2018

DOI: $10.23880 /$ apct- 16000141

\begin{abstract}
Pharmaceutical field encloses a wide number of themes from drug discovery to manufacturing and regulatory approval and involves several disciplines such as chemistry, biology, physics, biotechnology, pharmaceutics and engineering. In the last 40 years the interest and scientific literature about pharmaceutical research has rapidly grown. Indeed, the attention on public health has been increasing, probably due to the birth of many new diseases and allergies, problems associated to antibiotic resistance, more cases of cancer associated to environment and wrong lifestyles, etc. Therefore, which are the current trends in Pharmaceutical Research? For sure, to overcome the problems that have arisen in the recent years, new drugs and overall new drug delivery systems have been studying from public as well as private institutions. DDS carry and protect a pharmaceutical compound improving its administration and efficacy. Oral pills and injections are the most common formulations that are used nowadays. Since the oral and intravenous administration routes show some drawbacks such as poor bioavailability and noncompliance of the patient respectively, new alternative administration ways have been explored (i.e. pulmonary, transdermal, ocular, and nasal).
\end{abstract}

Keywords: FDA; DDS; Drug; Nanoparticles

\section{Introduction}

As result of this and thanks to a multidisciplinary approach, above all based on micro and Nanoscale, many novel DDS have been studied and some of them have reached the market. Those are transdermal, osmotic, mucoadhesive DDS, external medical devices and organic/inorganic Nano and micro particles [1]. Most of the studied and commercialized DDS are drug controlled release formulations that allow a slow delivery of the carried therapeutic in the time [2,3]. This leads to a reduction of administration frequency with a consequent gain in patient compliance and therapy cost decrease This is a depot system and consists of leuprolide encapsulated in PLGA microspheres and was approved by the FDA in 1989 for the treatment of advanced prostate cancer [4]. The main advantages of this system is the reduction of the daily injections of the drug alone to one every month (or every more months depending from the dose), PLGA biocompatibility and degradation, reproducible micro particles synthesis method. The research is advancing in this direction, mostly in finding novel materials and methods able to increase drug stability and protect it until reaching the target. Transdermals DDS are amply studied 


\section{Advances in Pharmacology and Clinical Trials}

because avoid the use of a syringe, further increasing patient compliance. These are basically composed by a protective backing layer, a reservoir (with the drug) and an adhesive layer to adhere to the skin. A dozen patches have been already approved by FDA among which Duragesic that carries fentanyl and is used for pain relief. From the problems that have arisen from the first generation of transdermal DDS, such as natural barrier properties of the skin, new techniques (ultrasound, microneedles, iontophoresis ) and materials (permeation enhancers has been explored to allow an effective transdermal drug permeation. Osmotic systems have been also attracting the researcher's attention for implantation as well as for oral delivery. OROS are examples of osmotic ally-driven DDS for an oral controlled release. These systems allow improving therapeutic time and concentration in plasma with consequent dose and side effects reduction as well as the administration of poorly soluble drugs. Actually the attention is based on the research and development of protective coatings for drug reservoir and in the use of mucoadhesive polymers to adhere to intestinal mucosa. Reservoirs implantable DDS have attracted a lot the attention above all for ocular drug delivery, because avoid numerous intravitreal injections. An example is Vitrasert approved by FDA in 1996. This is a reservoir system used for the treatment of AIDS-related cytomegalovirus retinitis that is able to release ganciclovir for a period of 5-8 months. In 2005 Retisert (fluocinolone) was approved by FDA for the treatment of chronic non-infectious uveitis reaching a very long-term drug release, up to 3 years. Recently, active release implants that can control the delivery of the drug by different methods (electrochemistry, laser, magnetic field and physical forces) are being designed and developed. Beyond drug controlled release systems, the research about external medical devices and nanosystems has also greatly increased. Metered dose inhalers for asthma or chronic obstructive pulmonary disease have been greatly studied as pulmonary DDS. The main advantages of these systems consist in an easy administration, absorption rapidity and the possibility to control the inhaled dose. Many devices have been approved and many others are being developed. One of the areas that were subjected to the fastest growing in the last years is the nanotechnology. Nanomaterial's such as liposomes, micelles, polymeric and inorganic nanoparticles show several advantages above all associated with their small size. In fact, those are able to overcome biological barriers, loading more than a therapeutic of different nature (i.e. hydrophilic and lipophilic) or with different function (i.e. a diagnostic and therapeutic drug to obtain a theranostics system) or interact with target tissue or cells thanks to a specific surface modification. Nevertheless, only few of these systems have reached the market due to the problems associated with the low accumulation into the target zone after injection or to not at all recognize toxic effects. Doxorubicin was the first marketed Nano system for the treatment of some cancers. This is a liposome that shows enhanced circulation and improved half-time respect to the drug alone. Doxil can target the tumor by enhanced permeability effect (EPR) [5]. This is a passive targeting that is suitable only for some types of patient; for other cases an active target is desirable. In current academic research, nanoparticles able to allow an active target have been studying as well as different type of nanomaterial's, from biodegradable, synthetic polymers to inorganic nanoparticles (such as iron oxide nanoparticles). The research in pharmaceutical field is continuously growing and covers a wide spectrum of disciplines. This let hope in meeting soon in the market novel formulations able to treat much more diseases improving public health as well as the patient compliance.

\section{References}

1. Malaterre V, Ogorka J, Loggia N, Gurny R (2009) Oral osmotically driven systems: 30 years of development and clinical use. Eur J Pharm Biopharm 73(3): 311323.

2. Robert C, Wilson CS, Venuta A, Ferrari M, Arreto CD (2017) Evolution of the scientific literature on drug delivery. J Control Release 260: 226-233.

3. Mitragotri S (2005) Healing sound: the use of ultrasound in drug delivery and other therapeutic applications. Nat Rev Drug Discov 4(3): 255-226.

4. Barenholz Y (2012) Doxil (R)- the first FDA-approved nano-drug: lessons learned. J control release 160(2): 117-134.

5. Maeda H, Wu J, Sawa T, Matsumura Y, Hori K (2000) Tumorvascular permeability and the EPR effect in macromolecular Therapeutics: a review. J Control Release 65(1-2): 271-284.

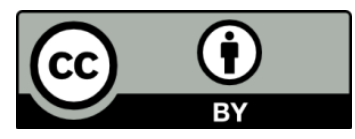

